\title{
Il Vocalismo tonico italiano.
}

I fogli, che seguono, sono la continuazione d' uno studio di fonetica italiana incominciatosi a stampare nella Rivista di filologia romanza, e rimasto poi in asso per la cessazione della Rivista stessa, alla quale sottentra la nostra Zeitschrift. Poichè la classe di lettori a cui si rivolgeva la Rivista non è precisamente la stessa a cui si rivolge la Zeitschrift, ho dubitato alcun poco della opportunità di proseguire qui la stampa del mio studio. Ne dò per ora solo i $\$ \S \mathrm{IX}-\mathrm{XI}$, che trattano dell' $\mathrm{E}$ lunga, accentuata; e insieme alla parte già publicata possono servire a dare un' idea di tutto il lavoro. Il quale sarà comunicato per intero, qualora $\mathrm{i}$ compagni di studio ne mostrino desiderio.

\section{$\S$ IX. - E lungo.}

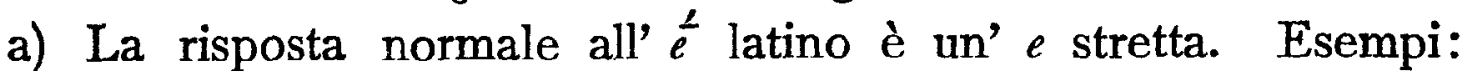
cannéto, pinéto, ulivéto, e gli altri in -éto = lat. -êtum; credéva, vedéva e sim. da credēbat vidēbat ecc.; avémo, vedémo da habèmus vidēmus ecc.; e così i futuri crederémo vedrémo, che si devono ricondurre a credere + habēmus, videre + habèmus e così via; vedéte ecc. da vidêtis ecc.; crederéte ecc. da credere thabètis ecc. Regolarmente $e$ stretta hassi anche in teméro (temèttero), credéro ecc., i quali devono essere ricondotti, più presto che alla base classica timuérunt credidèrunt, ad una di stampo regolare, foggiata su complēvērunt complērunt o su amavèrunt amarunt, cioè: timēvērunt timērunt ecc. Vengono poi le seguenti voci: candéla, téla, céla (cèlat), vélo (vèlum), véla (pl. vèla, vb. vēlat), léna aléna (nomi estratti da anhēlare con metat. alenare), réna (arēnam), avéna, véna, veléno, seréno, scémo (sèmis), séme (sèmen), céra (cêram), séra (sèram scilicet horam), véro, mercéde, créde, chéto (quiētum), tappéto (tapētum), réte, séta (sêtam 'setola' indi 'pelo'), acéto (acêtum), téga (thêcam), bottéga (apothēcam), méco, téco (mēcum ecc.), ségo sévo (sêbum), ecc. Aggiungansi i monosillabi accentati come ré (rēgem), tré (trēs).

Le eccezioni a questa legge sono di due specie. In alcune voci troviamo un $i$ schietto, scambio dell' $\dot{e}$; in altre, e sono ben più numerose, abbiamo un $\dot{e}$, ovvero $i \grave{e}$. Le prime sono: Messina

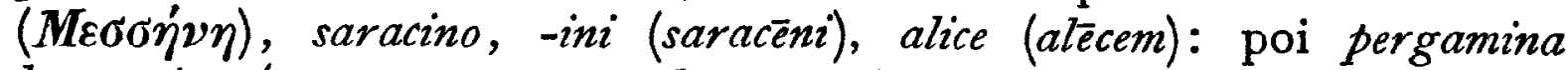
bergamina (pergamenam sc. chartam, in Isidoro pergamina), pulcino (pullicēnum), mantile (mantèle), tosc. volg. nimo (nēmo; nīmo in Donato 
secondo Schuchardt $I, 308$ ); infine berbice (vervēcem). - Le tre prime altro non saranno se non forme siciliane da raffrontare al serino, fice per seréno féce de' nostri dugentisti; e nel sicil. l' $i$ da un lat. $\grave{e}$ sta in piena regola, cfr. Arch. glott. II, 145: per Messina si potrebbe anche proporre, col Diez, la pronuncia neo-greca di

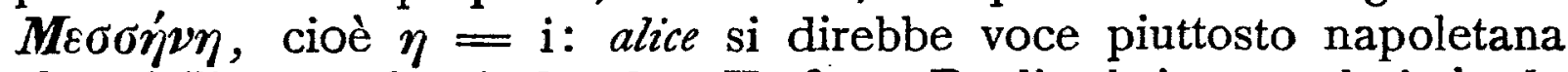
che siciliana, cfr. Arch. glott. II, 87. Degli altri esemplari è da cercare spiegazione diversa: poichè, se berbice potrà essere d' importazione francese, tutti gli altri sembrano di schietta formazione toscana, e ci riveleranno forse una peculiarità della pronuncia latina rispetto all' $\bar{e}$ tonico: cfr. il $\S \mathrm{XI}$ in fine.

Veniamo alle eccezioni dell' altra specie. - Abbiamo prima la serie di -ério che da -ièro -èro, invece di -ério éro; come si vede in mistèro (mystêrium), battistèro, cristèro (clystêrium), monistèro, fiera (fêriam), ciera (cêream scil. imaginem). Questo fatto, già notato dall' Ascoli, Arch. glott. I, 488, 495; II, 23, come si spiega? Diremo forse che, ad es., da féria s' ebbe prima férja, con l' e abbreviato dalla posizione romanza? Ma già dovetti negare assolutamente questa attitudine della posizione ad abbreviare le vocali latine. $\mathrm{O}^{\prime} l^{\prime} \bar{i}$ atono si sarà trapiantato avanto alla tonica: misteiro, $\mathrm{e}$ avrà operato su questa in via dissimilativa: mistéiro mistèiro, mentre in fiera e sim. passava innanzi all' $e$ per produrvi il dittongo raccolto? Tutte queste ed altre ipotesi tornano vane quando si tenga conto d' un' osservazione prosodica del Müller, De re metr. 359 . Egli ci avverte che $i$ poeti seriori calcolano breve la vocal tonica, a cui segua consonante semplice e un' $i$, che faccia iato: copiosior suffrăgium denărius, invece di copiōsior ecc. Adunque avranno calcolato anche mystêrium fëriam; infatto io trovo ecclësiam monastērium psaltèrium nella Sylvula enormium che il Browero raccolse dalle poesie di Ven. Fortunato (cfr. Opp. Romae I786). Ogni cosa così resta dichiarata; e féria fêria fëra dà il nostro fièra, come férum dà fièro ecc. Nello stesso modo và probabilmente spiegato dèbo dèva da débeo débeat attraverso débio débiat; mentre nell' arcaico diba dibia l' $i$ atona avrassi assimilato l' $e$ tonica. Altre eccezioni come: sincèro (sincērum), spèra (spērat), primavèra (cfr. vèr) (se pur è voce d' origine popolare), vanno pure spiegate, come indirettamente m' insegna l' Ascoli, Arch. glott. I, 275, da basi ipotetiche, quali sincerrium ecc., ossia come assimilazioni alle molte voci uscenti in -ěrium -ěriam. Nè si tralasci di notare che al tosc. sincèro ben risponde il ven. sinziero, e a spèra lo spiera raccolto dal Mussafia, Beiträge p. I I, ove pur si citano mistierio e gliesia: cfr. Arch. glolt. I, 327 in n., 331, 424. - Una seconda serie di eccezioni abbiamo in pièno quièto piève da plënum quiêtum plébem. Ma qui è facile notare, come già fece il Diez, Gram. I, 312, esser avvenuta un' assimilazione del dittongo $i \hat{e}$, sorto da $e ́$ ed $l$ vocalizzato, o da $i$ preesistente, col $i \vec{e}$, regolare risposta del lat. $e^{t}:$ in chilesa deve restar dubbio se l' iè sia sorto per questa via, o per quella che gli assegnammo in fiera ecc. 
Alcune altre eccezioni vanno trattate singolarmente. - Reda ridte per 'figlio', 'nato di bestia' non sarà probabilmente da herédem, ma, come ci dice la prima forma, dal nom. hereditas * rkida, con é allargato per dissimilazione: rilà erità $=$ hereditatem $\grave{\mathrm{e}}$ frequente nel veneto Buovo, edito dal Rajna. - Cede da cédit, concordando col veneto ziśde, accenna ad un' antica base popolare caedit o cédit: - rèmo (Diez, Gram. I, 312 rémo) da rémum ci ricorda il greco

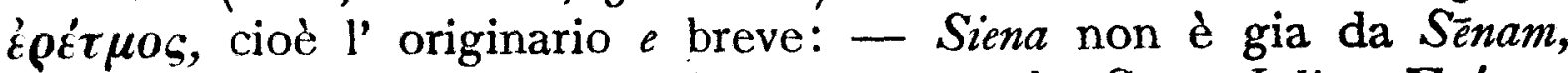

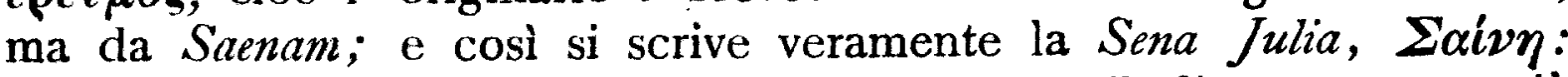
cfr. anche Schuchardt, $V_{0}$. I, 326: bieta deve il dittongo, non già all' $\bar{e}$ di bétam, ma ad $l$ inserito, od a quello del diminutivo bétulam, onde fecesi bletam; infatti blita sta nel Vocab. lat. berg., blide nel friul., cfr. Arch. glott. I, 315. Altri esempj come erede, crita, sede saranno da riguardare come appartenenti alla lingua dotta, e però regolari.

Infatto la lingua dotta e la semi-dotta attribuiscono il suono largo a tutti gli $e$ tonici, per la eccellente ragione che larghi $\mathrm{i}$ nostri dotti toscani proferirono e proferiscono gli $e$ tonici del latino. Qui stieno alcuni esempj sulla natura de' quali non possa esserci dubbio: problèma, teorèma, diadèma ecc., zèlo, collèga, lène, estrèmo, racèmo (cfr. racimolo), alèce (cfr. alice e aringa), complèto (cfr. compito), tutèla, parentèla, cautèla, decrèto, mansuèto, suprèmo, pergamèna (cfr. bergamina), Saracini (cfr. saracini) ecc. Alcune voci oscillano tra la forma popolare e la dotta, e così havvi chi dice crudéle e chi crudèle, chi fedéle e chi fedèle. Tra le voci semi-dotte possiamo ricordare strèmo per estremo, seguèla per sequèla ecc.

$\mathrm{E}$ qui ci occorre una importante osservazione. Le voci semidotte possono tanto esser prodotte dal letterato e poi accolte e modificate dagli illetterati, quanto anche venir create dal letterato secondo l' analogia del parlar popolare. Così, volendo usare il lat. examen extremus, il nostro letterato deve modificarli di tal guisa che $\mathrm{i}$ suoni, onde risultano le due parole, sembrino convenienti al tipo del dialetto fiorentino; e poichè questo non conosce l' $x$, e muta d' ordinario l' $x$ latino in $s$, i dotti da examen ed extremus fecero esame estremo. Questa osservazione ci spiega il perchè da noi si pronunci lauréto, pométo, frassinéto ecc. sebbene almeno la prima di queste voci sia indubbiamente di formazione letteraria. Gli esemplari della lingua comune, quali pinéto roveréto ecc., imposero la loro forma anche ai fratelli nati in modo meno legittimo della tradizione orale.

b) Abbiamo veduto come l' $\frac{\imath}{\imath}$ non s' ingrossi in $\dot{e}$ dinanzi a vocale ( $\$$ VII, $b$ ); e vedremo più innanzi come l' $\breve{e}$ dinanzi a vocale $\mathrm{s}^{\prime}$ assottigli in $i$ (cfr. dio da dëum): ragionevole adunque ci sembra l' ammettere che anche in solia vedia credia ecc., forme arcaiche per soléa vedéa ecc., soléva vedéva da solébat vidébat ecc., l' é italiano abbia volto ad $i$. Quando molti verbi in -êre vennero in tal guisa a confondere le loro flessioni anche in questo tempo usitatissimo con quelli in -ire (sentiva, veniva venia), poterono ben fare l' ultimo 
passo e accogliere anche $\mathrm{l}$ ' infinitivo in -ire invece di -ere. Così avemmo fiorire da florēere, pentire arc. pentére da poenitère, sorbire da sorbēere, olire da olère ecc. Ammettere collo Schuchardt un passaggio puramente fonetico da florère a fiorire parmi impossibile, stante il numero assai ristretto di esemplari italiani di $i=$ lat. $\dot{e}$. Bene del resto lo stesso linguista venne segnando a passo a passo l' accostarsi che facevano anche foneticamente $\mathrm{i}$ verbi in -ire ed -ere, rendendo così facile la fusione compita delle due forme. - L'Ascoli, Arch. glott. III, 72 n. 2 si domanda se la spinta prima a mutare l' $\bar{e}$ in $i$ non sia da cercare nel $c$ palatino che lo precedeva: cfr. il fr. merci, cire e plaisir, loisir, nuisir ecc. E il sospetto è pieno del solito acume. Un avanzo, vivo ancora almeno nella lingua poetica, di questo arcaico -ia per -ea -eva lo abbiamo ne' conditionali crederia sentiria, che vanno risolti in credere + avia, sentire + avia, come l' equipollente crederebbe in credere $+e b b e$.

c) Nella terzultima, ancora l' e stretta. Esempi: crédere, débole (dèbilem), débito, chérico (clèricum), sémina (sémina pl., sêminat vb.), fémina (fêminam), scévera, sépara (sēparat), sétola (sêtam), fécero (fécěrunt per fecērunt), quarésima (quadragēesimam).

Abbiamo $i$ per é in racimolo (racémum), a cui è da confrontare

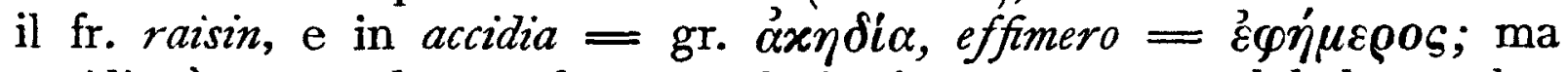
accidia è voce dotta, forse ecclesiastica, e presa dal basso-lat. accidia : effimero è voce semidotta, tolta direttamente dal greco, modernamente pronunciato. L' $\grave{e}$ per $\dot{e}$ ci appare per contrario in cèdere da cédere, sèrio: ed abbiamo già toccato de' così analoghi per la penultima.

La lingua dotta anche qui naturalmente ha l' $e$ larga; e citiamo a conferma: crèdulo, crèdito (cfr. créde), trigèsimo, millèsimo, centèsimo; mistèrio, saltèrio, monastèrio, sèrico; crètico, poètico, patètico, aritmettica, torpèdine. Tra le voci semidotte vanno poste: ventèsimo, trentèsimo, farnètico (frenéticum; ma il Cittadini, $O p p .203$, ha frenético, con forma interamente popolare). Notevole ci riesce céreo da céreum, voce dotta senza dubbio; ma che ebbe il suo $\dot{e}$ invece di $\grave{e}$, per influenza del popolare cèra da cèram.

d) Per la posizione spenta abbiamo numerosi esemplari di $\bar{e}$. Cicerone, Orat. C. 48 . $\$$ 159, ci avverte che "indoctus dicimus brevi prima littera, insanus producta, inhumanus brevi, infelix longa; et, ne multis, quibus in verbis eae primae litterae sunt quae in sapiente et felice, producta dicitur in, in ceteris omnibus breviter." cfr. Corssen I, ror. L' $n$ dinanzi ad $s$ fu sempre così fievolmente proferita dai Latini che taluno propose $d$ ' indicarla con un segno speciale, e di scrivere me:sis per mensis. La vocale antecedente, foss' ella breve o lunga per natura, restò sempre allungata da questa soppressione della nasale: e molto saggiamente il Diez considerò le vocali toniche, seguite da $n s$. quali lunghe ordinarie, avendosi p. e. in ital. mése da mensem (me:sem) come créde da crēdit. - Citiamo: pésa (pensat), spéso (expensum), offésa (offensam: 
cfr. il dotto offensa), teso (lensum), accéso (accensum: cfr. incènso, voce lotta da incinsum); indi: Parmése (parmēnsem) Sanése Ateniése ecc.

Del resto per me resta ancor dubbio se in pensat, offensa ecc. l' e fosse naturalmente breve, come de in pëndere offëndere, o se sia già stato allungato dalla soppressione d' una consonante, stando offensa per offénd-sa ecc. Certo in mensis, cfr. $\mu \eta^{\nu} \boldsymbol{\mu} \mu \nu \boldsymbol{\nu}^{\prime}$, l' $e$ era lunga, anche prima che l' $n$ susseguente venisse fognata; e le trascrizioni greche ci consigliano la stessa ipotesi rispetto ai nomi in -ensis. - Tuttavia i riflessi romanzi di pensare nel senso di 'co-gitare' ci mettono qualche dubbio sulla quantità originaria del suo e: cfr. lo spagn. piensa. Esempi dubii di posizione spenta sono gli arcaici rei lei per 're' 'legge', che potrebbero risalire a réx le $x$ (cfr. régem légem), come sei a sex. Ma chi ci assicura poi che non sieno provenzalismi, oppure anche evoluzioni regolari nostrane di rélg)em legem?

In pisolo (- pensilem?) e nel toscano volg. tiso per teso, l' $e$ stretta è proceduta sino ad $i$.

Voci dotte qui naturalmente non vi possono essere, poichè $\mathbf{i}$ dotti, attingendo al lat. scritto, conservarono quell' $n$, che pur era in gran parte svanito già nell' età del buon latino: e così dissero offènsa, dispènsa, incènso, accènso, pènsile ecc.

e) Sempre lo stesso esito. Esempî: légge (lègem), Arézzo (Arētium), rézza rézzola (rētia pl.), vénne (vènit pf.), scélto (sēlectum), crébbe (crēvit), érto (èrēetum), bélva.(béluam, cfr. Grazio Fal. v. 88), sézzo arc. (sêcius); nelle flessioni verbali: compiémmo vedémmo avémmo, che risalgono a complè(vi)mus, *vidè (vi)mus, habé(vi)mus, forme ridotte regolari, dai temi vidé- habé-, sull' analogia di amammo da $a m \bar{a}(v i)-$ mus: lo stesso si dica dei condizionali crederemmo, amerémmo ecc., che vanno risolti in credere + avémmo.

All' $i$ schietta arriva l' $e ́$ da $\bar{e}$ in quitto da quiêtum, forse per assimilazione coll' $i$ radicale.

Abbiamo invece l' $e$ larga in camèllo (camélum), visibilmente assimilato ad agnèllo porcèllo ecc., e in dèbbo dèbba, forme analoghe ad altre di cui già discorremmo.

Alla lingua semidotta attribuirei èbbro èbro da èbrium, mentre puramente dotto è èbrio.

f) Esiti uguali: tégghia (têgulam), scéglie scélgono (sêligit sêligunt), fémmina, vendémmia (vindēmiam), bestémmia arc. biastéma (blasphèmia).

Fanno eccezione il solito dèggio dèggia, ed èrgere, cfr. érto, se pure non è un error del Fanfani; ma sarà forse pronuncia dotta d' un popolare érgere, ornai svanito dalla tradizione parlata.

g) Sempre é. Esempî: vénde (vēndit, cfr. vēnum), ésca (ēscam, cfr. èsus prcp. di ědere ed-tus), crésce (crèscit, cfr. crēei crètum), têtto (tēctum, cfr. tēgula, di fronte a tĕgere: Schuchardt, Vok. I, 333), régno (rēgnum, cfr. règem); nelle flessioni verbali: compiésti, avésti, temésti ecc. da complèsti $=$ complèvisti, e analogamente *habé(vi)sti, * timé(vi)sti, indi compiésse, avésse, facésse da complèsset complé(vi)sset, 
e analogamente habe(vi)sset, facé(vi)sset. - Notevole esemplare è prende da prěhendit o praehěndit prēndit: il Cittadini, Opp. 2 I I, proferisce prénde, il Fanfani prènde; e ciò vorrebbe dire che nella evoluzione italiana di prëhěndit s' ebbe da un lato fusione delle due vocali in una lunga: prēndit, dall' altro esisione: prëndit o praendit.

Non veggo esempî di $i$ da $\bar{e}$.

Un è per $e$ abbiamo invece nel solito tema cêd-: cèsso, concèsso, concèsse; in protèsse, dirèsse ecc. da protexit, cfr. lëgere, rĕgere, se pur è attendibile l' osservazione di Prisciano (cfr. Schucharett. Vok. III, 136), che afferma essersi pronunciato texxit, rexxit; ma fino a prova migliore noi crederemo nella brevità di questo $e^{\iota}$, o ammetteremo un' assimilazione italiana delle forme del perfetto, relativamente rare, a quelle del presente, dove l' $\grave{e}$ dà $\breve{e}$ riesce legittimo. In pènsa da pensat èvvi eccezione? Lo spagn. ha piensa, che risale regolarmente col nostro pènsa ad un pën-sat, cfr. pëndere.

Nelle voci dotte e semidotte hassi $e$ larga: incesto (cās-tus in Schuchardt I, 369), recèsso, incèsso, mènsa (mēnsam, cfr. mêtiri: mensa fu veramente la 'porzione', la 'portata'), immenso, scittro ( $\sigma x \tilde{\eta} \pi \varrho o \nu)$, plettro $(\pi \lambda \tilde{\eta} x \tau \rho o \nu)$.

h) Egualmente: véndere créscere; compiéssimo avéssimo ecc. da complè (vi)ssemus ecc. con accento rattratto, complèssémus ecc.

In minchia da mentulam ( $e$ lungo?) l' $e$ stretto, che si ha nel doppione ménchero, si riduce ad $i$ schietto: forse par influenza del deriv. minchione.

Le voci dotte con è: rèttile (cfr. rēpere), acquièscere (cfr. quiētus) ecc.

$\S$ X. - E breve.

a) L' $\mathrm{e}^{\prime}$ breve latino ha qui per risposta regolare $i \grave{e}$, più raramente $\mathrm{e}$ in determinate circostanze $\dot{e}$. Citiamo con $i \grave{e}:$ dieci $($ dĕcem), diede (dëdit), ieri (hĕri), fiero fiera, lieve, piede piè, vieto (vĕto vb. e vêtus agg.), riede (rēdit), tiene, siede (sëdet), fiele, miele, miete (mētit), siero arc. sielo (sêrum), arc. nievo (nëpos), mestiere (ministèrium); quindi, con forma oscillante fra $\grave{e}$ ed $i \grave{e}:$ prèga priega (prěcat-ur), brève brieve, prème prieme, trèma triema, lèva sost. e vb. lieva (ma sempre allievo, nome estratto da allevare), gème gieme, gèlo gielo, èra iera (ĕrat); infine, solo con è: béne (bêne) (ma il Cittadini ci avverta che i Perugini dicono biène; e il dittongo appare in altri dialetti italiani e nel fr. bien), crèpa (crëpat), frème (frëmit), che forse non è popolare, scèda 'mostra' (schëdam) e forse altri.

Queste due ultime serie di esempî ci mostrano che l' $\dot{e}$ è preferito ad $i \grave{l}$ dopo un $r$ complicato, quasi a togliere le difficoltà della pronuncia.

Notevoli sono poi: siègue ségue, nièga néga, séga trevig. siéga (sếcat); ove il dittongo alterna, non più coll' $e$, ma coll' é. Pare evidente che ciò si debba alla qualità della consonante seguente, $g$, $g u$ : che, come nel famoso esemplare aqua aigua, avrà trasmesso 
all' $e$ tonico un elemento $i$, per l'influenza assimilativa del quale, $l$ 'e, già stretto, come vedremo, in sequilur, si mantenne invariato. Noto di passaggio che $i$ Veneti dicono: siguita $=$ it. séguita ma poi niga siga coi Toscani.

1 Toscani leggono con suono aperto l' $\bar{e}$ tonica latina; e però i naturale che nelle voci dotte esso abbia conservato questo suono; valgano ad esempio: geme, mèro, gèna ecc.

b) Nelle voci popolari l' $\breve{e}$ qui si assottiglia in $i:$ dio (dêum), mio (mẽum), mie (mĕae), rio (rêum), arc. cria (crĕat); e così pure avviene, se l' $\breve{e}$ si trova dinanzi a vocale, per la soppressione d' una consonante: io arc. eo da $\ddot{e g o}$, scia da sëcat, cfr. il fr. scier scie da secare. Sicia è per noi il 'solco della barca sull' onde'. Notevole è mièi da mễi.

Nelle parole della lingua dotta si mantiene l' e latina: rèo, crèa, dèa $(d \bar{e} a m)$, e il plur. dei (dêei, non $d \hat{\imath})$ ch' è pieno di significato, in quanto ci dimostra come il Cristianesimo abbia cancellato dalla memoria del popolo nostro quella folla di celesti, che dominavano l' olimpo pagano: il plur. iddii è formato recentemente dal singol. iddio. - E il comunissimo idèa è di formazione popolare? Non parrebbe dalla sua forma; ma non è da dimenticare che la voce è greca: i $\delta \varepsilon ́ \alpha$, e che i Latini la pronunciarono forse idea. Ovvero nella tradizione popolare si sarebbe perduta questa voce esprimente una funzione superiore?

Nell' $i$ di mio dio ecc. vollero taluni scorgere la continuazione del lat. arc. mius dius; ma giova notare, col Diez, essere tale ipotesi inutile: oltre che riescirebbe difficile in essa a spiegare il miei.

c) Con iè: miètere, tiepido tosc. chiepido (tĕpidum) daccanto a tèpido, diedero (dëderunt), arc. stiedero (stëterunt), siedere (sëdere), fiedere fierere (*fêrere per ferire), Nievole n. loc. (Nëbulae); e nella terzultima, sorta per l' evoluzione italiana: vietano siedono mietono ecc. - Con è; tènero tosc. tèndero, gènero (gēnerum), mèdico (mëdicum), mèrito (mëritum: il Diez, Gr. I, 3II, per errore mérito), rèdine (* rëtinae, nome estratto da rētinere), mèliga (mëdicam sc. herbam), ripètere (re-pĕtere); indi matèria, misèria, desiderio, macèria, spècie spèzie, schèletro arc. scheltro ( $\sigma x \varepsilon \lambda \varepsilon \tau o ́ s)$, voci che non mi hanno fisonomia schiettamente popolare.

In édera ellera (héderam), e in prezzémolo petrosémolo ( $\pi \varepsilon \tau$ ¿0$\sigma \varepsilon \lambda_{\iota v o v)}$ hassi l' $e$ stretta: noto che il trevigiano ha erola. $-\mathrm{L}^{\prime} i$ schietto appare in risica rischia da résecat (Diez, Voc. et. I, 352) dove s' ebbe l' $i$ dapprima nella vocale atona: risicare da resecare come ricevere ricoverare ecc. da recipere ecc., e fu poi trasferito alla tonica; e nel tosc. volg. nivola per 'nuvola' da něbulam, ove non saprebbesi ben dire se l' $i$ sia nata dalla fusione di ie (cfr. quitto da quiètum), nievola nivola, o se direttamente siasi assottigliato, come farebbe credere nébbia e il trevig. nibia; indi negli arc. benivolo, malivolo, ibano.

Le voci dotte vanno naturalmente con $e$, e non possono quindi per questo verso distinguersi dalle popolari: lepido, imperio, pelago, 
tripido, tremulo, cripita (crëpitat), cèlebre, cellere, stèrile, vènera, Vènere, genio, sèrie, e fors' anco gènere ecc. Altri esempi dubbii furono citati più in su.

d) Nella posizione spenta l' $\breve{e}$ si comporta come dinanzi a consonante originariamente scempia: Piero Pietro; prièta (pētram); dirieto (de-rëtro); intièro, ma poi intéro, col dittongo probabilmente fuso in un sol suono, da intĕgrum, romanamente intégrum; diero che tanto può esser venuto da diedero (dêderunt), escluso il $d$ me-

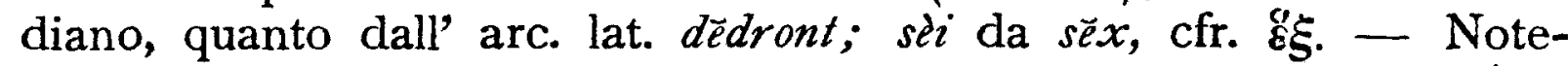
vole esemplare è prète arc. preite priete da prēsbyter, gr. $\pi \varrho \varepsilon \sigma \beta v ́ \tau \varepsilon-$ pos: qui l' $s$ era già caduto per tempo nel lat. volgare, almeno in molte regioni, poichè l' ital. ant. conosce anche presto, e l' ant. franc. prestre. L' $\dot{e}$, insieme coll' arc. $i e$, ci rende qui l' $\bar{e}$ del tema? ovvero s' ebbe prébyter, con $e$ allungato per compenso, e di là prévite, préite, prè(i)te, per dissimilazione?

La prima ipotesi ha, parmi, maggiore probabilità.

e) Qui per norma l' è. Esempî: venngo vègno ecc. (vënio), tèngo tègno ecc. (tëneo), lèpre (lëpörem), lègge (lëgit: cfr. légge da légem), règge (rëgit: cfr. ré rège da rēgem), sèggo (sêdeo), sèrra (sêrat), mèrlo (mêrulum), gèrla (gěrulam), mèlliga (mëlicam medicam), soffêrto offerto ecc. da * sufféritum ecc., cfr. fëro, grèmbo grèmbio (grëmium). rimèmbra (re-mèmorat), arc. insèmb̈re (in-sēmul).

Fanno eccezione con é:-ingégno (cfr. il fr. engin) da ingĕnium, e secondo il Fanf. grégge gréggia (grégem: secondo il Diez, Gr. I, 3 I I grìgge); e vi sospettiamo influenza della posizione palatile. L' eccezione di grémbo (grèmium), messa innanzi dal Diez, ibidem, non sussiste, essendo comune e regolare la pronuncia: grembo.

f) L' ̀̀ normale: lèggere, règgere, sèggio (sĕdeo), vècchio (vètulum), spècchio spìglio (spëculum), vèngono tèngono ecc.

Fann' eccezione nébbia da nébulam, éllera da hẽderam, di cui fu già discorso.

Mancano voci dotte.

g) Per lo studio dell' $\bar{e}$ in posizione latina di penultima abbondano gli esempî: e mette conto di farne un' ampia rassegna;

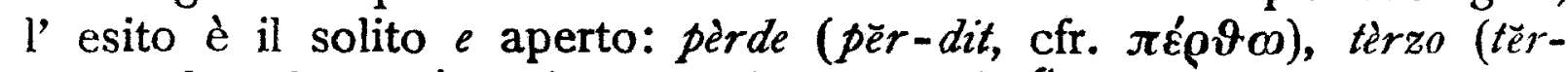

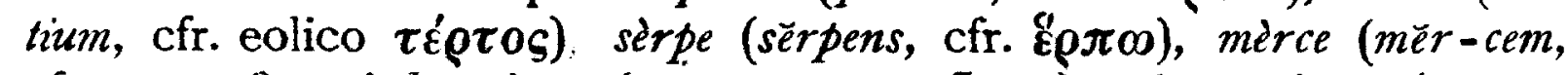
cfr. mëreri), nèrbo nèrvo (nërvum $=\nu \varepsilon \bar{v} \varrho 0 \nu)$, sèrvo sèrve (servum servit, cfr. $\sigma \varepsilon \dot{\rho} \beta \mathrm{s}_{\mathrm{S}}$ in Lydus, De mag. I, II, 129), govèrna (gubernat,

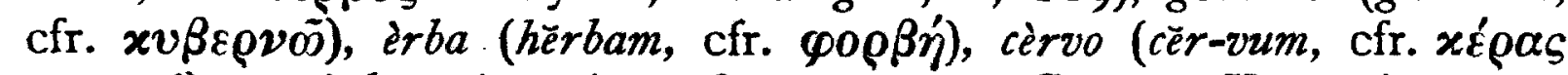
'corno'), supèrbo, verso (supērbum, vērsus: Corssen II, 245), verrme (vërmen, cfr. sanscr. krmi: Ascoli, Fonol. 69-70), tavèrna (tabërnam :

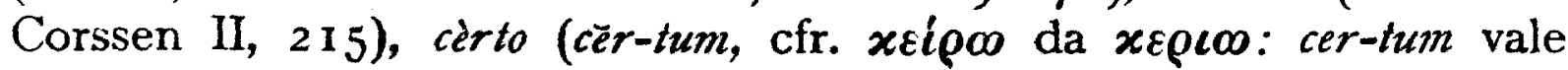
'tagliato' 'deciso' 'preciso'), desèrto (desertum, desêro), apèrto (apērtum apërio), copèrto (coopër-tum coopërio), invèrno (hibër-num scil. tempus: cfr. Ascoli, Fonologia I, 178), terra (têr-ram per ter-sam, cfr. $\tau \dot{\varepsilon} \rho-\sigma o \mu \alpha \iota$ 'inaridisco': terra è la parte 'secca', il continente, rispetto al mare); gente (gentem, cfr. gènus), rènde (rēd-dit), dènte

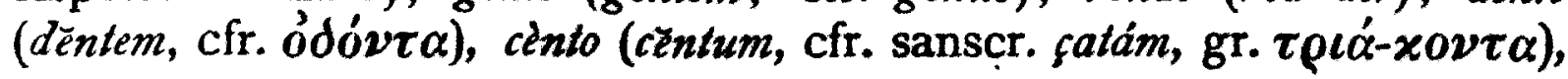


findi (pindit: Corssen I, 103), difende (defëndit, cfr. છgivo per Orl'(1)), linde (lindit, efr. $\tau \varepsilon \dot{\imath} \boldsymbol{\nu} \omega$ per $\tau \varepsilon \nu(\omega)$ ), ascende discènde (scăndit - sanscr. skíndati: Ascoli, Fonol. 31), talento (talëntum: Corssen II, I10), tènta (tën-tat, cfr. tëneo), contènto (contën-tum), vènto convènto (vën-tum, cfr. vënio), mente (měntit-ur, cfr. mën-tem), esènte esento

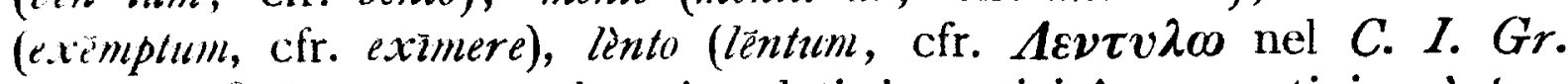
2943). Qui vanno anche ricordati i participî presenti in -ente e $i$ gerundî in -èndo, che risalgono ad -èntem -êndum: in venientem reniendo $l$ ' $\bar{e}$ breve per natura ci è rivelata, oltre che dalle trascrizioni greche quali ó $\psi \varepsilon \boldsymbol{x} \boldsymbol{0} \boldsymbol{v} \boldsymbol{v} \boldsymbol{\tau} \varepsilon \boldsymbol{G}$ in Plutarco, De fort. vir. 322 ecc., anche dal confronto dei partic. greci, quali $\pi o \iota \varepsilon-o ́ v \tau \alpha$, o $\tau \iota \xi \dot{\varepsilon} \nu \tau \alpha$; $\mathrm{e}$ le trascrizioni vogliono breve anche l'e di dolentem timentem e

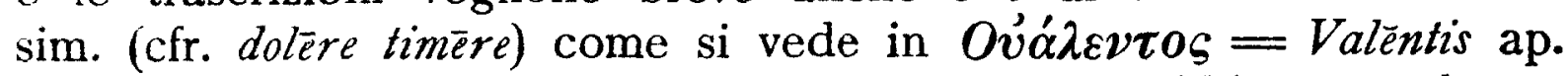
Corssen II, 250: e potrassi quindi chiedere se abbia avuto luogo un' elisione: valēelèntis = valëntis; ovvero, se veniëntis credëntis e simili, i quali naturalmente avevano l' $\bar{e}$, si abbiano assimilati gli altri. - Vengono poi: gèlso (cëlsum, cfr. per-cüli), velttro (vertragum,

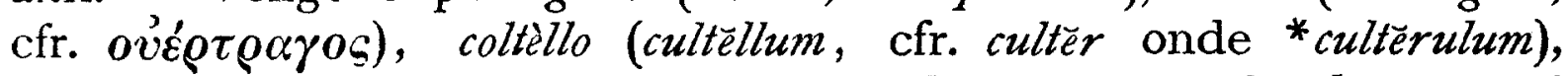
(fagęllum, cfr. flagrum = flagěrum), belllo (bellum da běne quasi benulum), vitello (vitūtum onde vitêllum), tosc. volg. cavelle covelle (quod- quae vēlles, cfr. vōlo), gemèllo giumèlla (gemëllum, cfr. gemīnus, onde il dimin. * gemīnulum = gemellum), fratèllo (* fratêllum, cfr. fratēr) ecc.; novembre settèmbre (cfr. novëm septëm), tèmpo tèmpra (tempus temperat, cfr. $\tau \varepsilon \dot{\mu} \mu \nu \omega)$, mèmbro arc. vèmbro (membrum, che starà probabilmente per *me-merum, cfr. $\mu \varepsilon \dot{\varrho}$

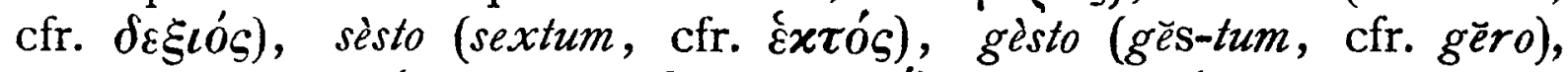
pèsca per persca (përsicam, cfr. $\left.\pi \varepsilon \varrho \sigma \iota x \eta^{\prime}\right)$, èsce èsca (ex-it ex-eat, cfr. ¿̇ $\xi$ : e paragona ésca da ês-cam), finèstra (fenèstram: Corssen II, I I4), tèsta tèsto (tër-tam dalla rad. tër- onde move pur terra; testa val pertanto 'terra cotta', 'pignatta' indi 'capo', 'teschio'), Trièste

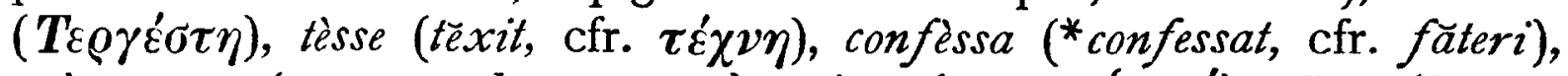

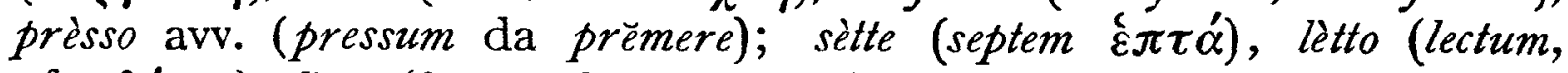

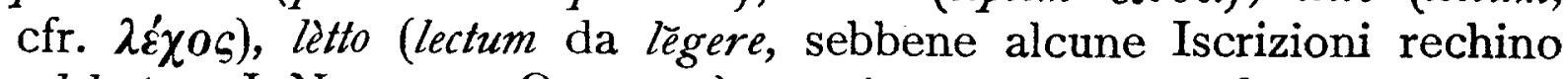
ad-lēctus: I. N. 1999; Or. 4109), protètto (protectum, cfr. těgo), dilètto (cfr. delĩciae), nèzza fr. nièce (* nëptiam, neptis, cfr. nëpos), pècca (pĕccat quasi pët-icat, rad. pet- 'lanciarsi', 'volare', 'piombare,: così da siticat è siccat), ècco (ëccum, cfr. ën). - Nella posizione debole di tr può svilupparsi anche il dittongo, come si vede in: Pietro pietra, dietro (de rĕtro); ma dall' altro canto hassi febbre, cèdro (cĕdrum).

Ore tenteremo di toccare almeno le più importanti eccezioni alla legge dell' $\grave{e}$ da lat. $e^{\iota}$ in posizione. $\mathrm{E}$ ci si offrono per primi tutti gli avverbi in -ménte, insieme collo stesso sost. ménte da méntem (sanscr. manas, e cfr. mëmīni). E come diciamo: chiaraménte feliceménte ecc. diciamo anche giuraménto, moménto, torménto ecc. quantunque il suffisso lat. mentum vada risolto in mën + to. Come ci spiegheremo queste deviazioni da una legge che pur riscontrammo cosi sicura in un' ampia serie di esemplari? Dubiteremo forse della quantità di men- in mentem -mentum, osservando che mën e 
-mōn- in -mōnium (patrimonium ecc.) sono identici originariamente? A questa ipotesi s' oppone recisamente lo spagn., ove le voci popolari hanno il dittongo nel suffisso -mentum: deleitamiento, fallamiento, sufrimiento, e anticamente l' ebbero anche negli avverbi composti di -mente: sutilmientre, bellamientre, mentre la lingua moderna usa solo -mente (Diez, Voc. et. I, 272). Nello spagn. è facile vedere, anzi tutto, l' influenza della lingua letteraria, che rifoggiò le parole popolari antiche sullo stampo latino: la tradizione orale però ivi risale senza dubbio a mëntem e -mëntum, poichè lo spagn.

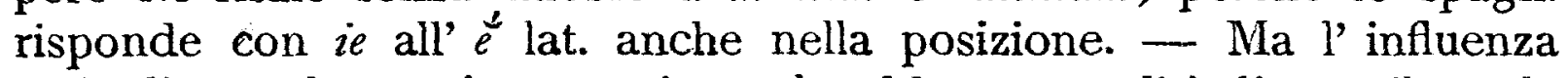
della lingua letteraria non si potrà addurre per l' italiano, il quale ci mostra $e$ specialmente nelle voci dotte. - Diremo infine che l' $-n t$ - abbia trasmesso un sottile elemento di $i$ (cfr. $\S$ VII g) all' $\breve{e}$ tonico, così da impedirgli l' evoluzione ad $\grave{e}$ ? Nulla di tutto questo ci sembra accettevole: e, fino a migliore spiegazione del fatto, noi proporremmo di ammettere un puro istinto differenziativo tra ménte da mëntem e mènte mènto da mëntiri: differenziamento che si allargò poi anche agli avverbi in -mente $\mathrm{e}$, ai nomi in -mento. - Altre

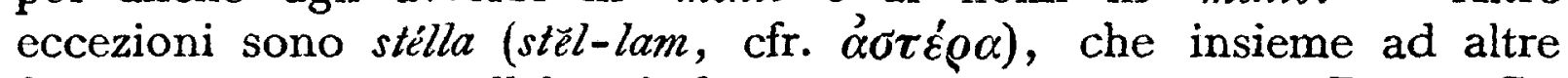
forme romanze parallele ci fa supporre un lat. stêla: Diez, Gr.

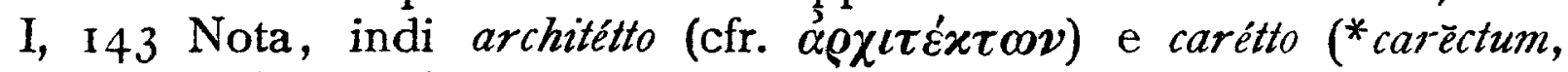
cfr. carĩcem), che furono tutti e due assimilati a i tanti nostri diminutivi in -étto (palazzétto ragazzétto), e il primo fors' anco a tétto. Le eccezioni proposte dal Diez, Gr. I, 3 I I, quali: témpio témpia, rénde, ménto (mentior) ecc. non sussistono, pronunciandosi queste voci con $\grave{e}$.

Notevoli sono alcuni esemplari con $i$ schietto al posto dell' $\grave{e}$ regolare: ritto di-ritto (rëctum, cfr. rĕgo), profitto (proféctum, profício), gli arcaici respitto despitto (-spèctum, cfr. spëcio); indi gitta tragitta $\left({ }^{*}\right.$ jectat trans-jectat, cfr. jăcio), e infine registro (regěstum, regēro). Per tutti non può valere una stessa dichiarazione: diritto non sarà dal lat. class. dirěctum, ma da un nuovo part. dirictum, coll' $i$ del pres. diř̆go mantenuto: ritto sarà assimilato a diritto; $\mathrm{e}$ così profitto da profictum, per profectum, forse con assimilazione a fitto (= fictum) 'il censo', 'la rendita che si ritrae da un fondo'. L' obbiezione che anche da profĩctum dirĭctum si aspettava profétto diréito (cfr. $\S$ VIII $e$ ) non vale, trattandosì qui di un $\check{z}$ tonico di formazione seriore, più italiana che latina. - Rispetto a respitto ecc. deve restar dubbio se sieno forme sorte per simile via, o se sieno francesismi, o infine provincialismi italiani. - Degli altri esemplari non so veder altra ragione, che quella sospettata già dall' Ascoli, Arch. glott. III, 72 n. 2: l' influenza, cioè, della conson. palatina, che precede.

Le parole dotte e semi-dotte hanno regolarmente l'e larga, e possiamo citare a conferma: esterno (extērnum, cfr. extërus), superno (cfr. supērus), intèrno, progresso regrèsso ingrèsso (cfr. ingrëdior), spèttro, fesso 'stanco' (fessum, cfr. fătisco), gèrme (gërmen, cfr. gēnus), sètta (sêctam, cfr. séquor o sĕcare), infetto perfelto prefetto ecc. (cfr. 
facio), ecilso (ex-çlsum, cfr. $x \xi \lambda \lambda \varepsilon \iota v=-$ cellere) ecc. Si possono aggiungere non pochi aggettivi in -èsto -estro -estre come modesto, rubesto, celeste, terrestre, campistre, silvestre ecc. poiche i Latini proferivano breve l' e di quest suffissi: cfr. Schmitz, nel Museo Renano XI, OIf segg. - Alcune voci dotte hanno l' $e$ stretta per ragione di assimilazione: e così diciamo emoluménto monuménto ecc. e lautamínte ecc. perchè queste parole o tolte dal latino o create analogicamente dai dotti, avevano dinanzi a se il tipo popolare con $e$ stretto, come già avemmo a notare.

h) Esiti uguali: persico, pèrdere, rindere, termine (terminum, cfr.

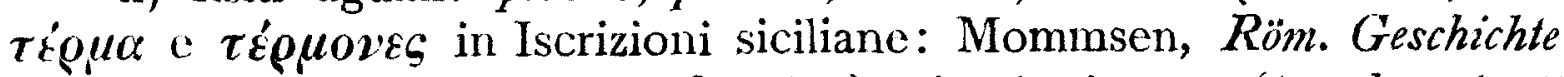

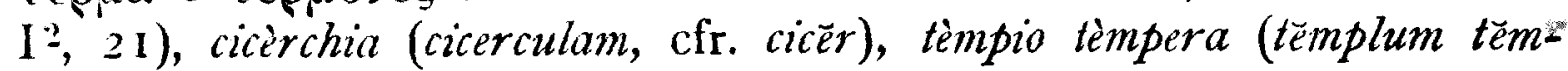
perat, cfr. $\tau \varepsilon \dot{\varepsilon} \mu \varepsilon v o s:$ Mommsen, l. c.), teschio (*tesstulum da těs-tu),

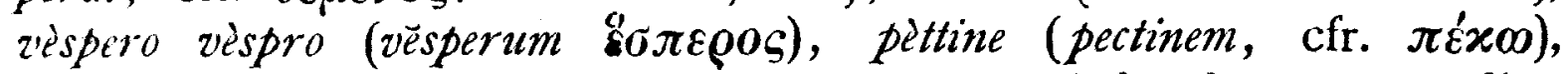
piggio (péjus che sta per pes-jus, per-ius, ed ha la stessa radice di pessum-do, pestis ecc.), èssere (ësse, cfr. èram čatí).

Le voci dotte, anche esse con $\ddot{\imath}$ : estero $(\check{e} x$-terum, cfr. $\stackrel{\varepsilon}{\xi} \xi)$,

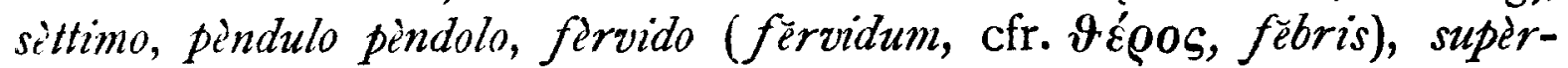
stite (supir-stitem), esercito (exér-citum: Corssen I, 316), lèssico $(\lambda \varepsilon \xi(x o ́ v)$, èstasi $(\dot{\varepsilon} x-)$, èccita (ex-citat), celebèrrimo, salubèrrimo ecc.

\section{$\S \mathrm{XI}$.}

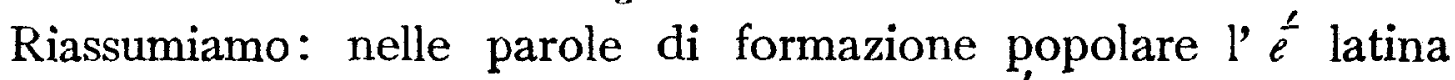
trova la sua normale risposta in un $e^{2}$ italiana, l' $\grave{e}$ in un $\grave{e}$, la quale dinanzi a consonante scempia, specie nella penultima, può spezzarsi in $i \grave{e}$. Nelle parole di origine dotta o semidotta invece hassi $z$ tanto da $\breve{e}$ quanto da $\bar{e}$ : e questa ultima legge fu già riconosciuta dal nostro Cittadini, $O p p$. r 99 .

Ora, prima di cercare come il nostro popolare $\grave{e}$ sia venuto dal

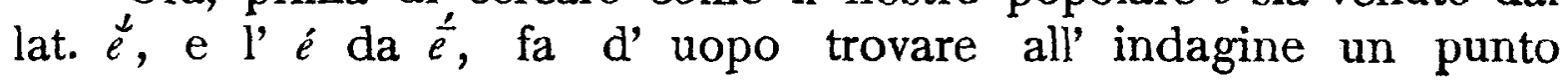
d' appoggio nella determinazione della pronuncia del lat. $\bar{e}$ ed $\bar{e}$ tonici: la non è questione agevole. Un tardo grammatico ci avverte che l' $e$ 'quando productum est, sic sonat quasi $i$, ut deméns' (cfr. Schuchardt, Vok. 1, 226 e segg.); e che 'quando correptum est, sic sonat quasi diphthongus, équus' (ib.). - Questa affermazione di Sergio, e i tanti esempii di forme basso-lat. con $i$ per $\vec{e}$, e $a e$ per $\breve{e}$, raccolti dallo Schuchardt, ci provano solo che l' odierna pronuncia italiana, astrazion fatta dal dittongo $i e$ per $\grave{e}$, ci rappresenta uno stato relativamente antico dei suoni d' $\bar{e}$ ed $\bar{e}$; ma non ci provano punto che questo rapporto sussistesse fino dall' epoca del lat. classico. Anzi, se badiamo a doppie grafie, quali sono scaena

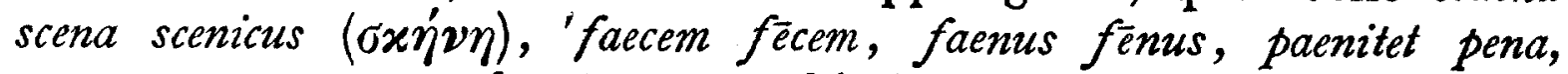
caespes cespes ecc., dovremo conchiudere che l' $\bar{e}$ suonasse molto simile all' ae, cioè largo: nella quale opinione siamo confortati dal fatto che gli esempî citati da Quintiliano I, 4, 8 di $\bar{e}$ proferita $\dot{e}$, sono tutti fuori d' accento. Come pertanto ammetteremo che in generale l' $\frac{6}{e}$ classico suonasse aperto, cosi dovremo ammettere che il classico $\mathscr{e}$ suonasse chiuso, considerando come esso, in moltissimi 
casi, altro non sia che un $i$ oscurata: perféctus da perficere, corrëptus da corräpere, dirěctus da dirigere o anche praecēps dallato a praecipitis, judex dallato a judicis ecc. - Se queste due induzioni sono bene appoggiate, ogni cosa si rischiara da se. Infatti per le leggi fisiologiche, che cercammo di stabilire nel $\S \mathrm{VWI}, \mathrm{l}$ ' $\bar{e}$ larga di credit per tramutarsi nell' e stretta di créde, ha dovuto abbreviarsi: e l' $\breve{e}$ stretta di béne per tramutarsi nell' e larga del nostro bène, ha dovuto accorciarsi. Di quanto? $\mathbf{E}$ facile rispondere ormai. Poichè l' uno e l' altro hanno percorso in senso inverso la distanza ch' è tra $\grave{e}$ ed $e ́$, distanza che giudicammo di mezzo tuono, è necessario conchiudere che l' uno siasi accorciato e l' altro siasi allungato della stessa quantità, cioè di mezza mora. $E$ in fatti

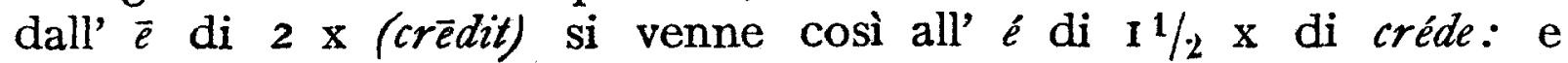
dall' $\breve{e}$ di uno $\times$ (bêne) si arrivò all' $e$ di $1 \frac{1}{2} \times$ nel nostro bene: chè $\mathrm{l}$ 'e di créde e béne hanno per noi uguale durata.

A mezza mora pertanto di quantità mutata, risponde mezzo tuono di qualità mutata. - $\mathrm{E}$ così benissimo ci spieghiamo anche in qual modo le risposte italiane del lat. $\frac{\dot{t}}{e}$ ed $\frac{1}{2}$ abbiano dovuto incontrarsi e confondersi nella nostra $e$ stretta. In effetti, tra l' $e$ larga e l' $i$ c' è un tuono intiero di distanza, diviso in parti eguali dall' $e$ stretta. - L ' $\frac{1}{2}$ latina s' allungò di $1 / 2 x$, passando nell' italiano, e s' ingrossò in conseguenza di mezzo tuono, diventando $e ́$ (néve $=$ nĩvem): l' $e^{\frac{1}{e}}$ latino s' abbreviò nell' ital. di $1 / 2 \mathrm{x}$, e si restrinse perciò di 1/2 tuono, diventando $e ́$ anch' esso.

Vediamo se questa spiegazione sia applicabile anche ad altri linguaggi neolatini: consideriamone l' azione per entro il dialetto siciliano (palermitano). Qui da lat. $e^{\frac{1}{}}$ ottiensi un $i$, da lat. $e^{\grave{L}}$ un $e^{\prime}$ : catina $=$ catēnam, téni $=$ tènet. L'è lat. di catēnam, per giungere all' $i$ di catina, ha dovuto percorrere la distanza di un tuono intero, e però abbreviarsi d' una intera mora: l' é di tênet, per conservare, il suo suono stretto originario, ha dovuto conservare l' originaria sua quantità, di una mora: e così anche nel siciliano la durata delle due toniche fu conguagliata, ma non più al $1 \frac{1}{2} \times$ fiorentino; ma ad un $x$ puro e semplice: la tonica siciliana ci dà l' esatta misura della breve latina. - Teoricamente così giungiamo ad affermare che la tonica italiana (toscana, fiorentina) è più lunga della siciliana di $1 / 2 \mathrm{x}$; e la pratica avverte anche $i$ non filologi che il siciliano pronuncia le vocali toniche più brevi del toscano. Quelli interrogati da me farebbero la tonica palermitana di circa un terzo più breve della toscana.

Ho condotto questa ricerca per una via un pò intricata, partendo dal fatto solamente probabile che il lat. classico proferisse largo l' $\bar{e}$ tonico e stretto l' $\breve{e}$. Ma ora che la materia parmi dilucidata, potremmo anche partire: $\mathbf{I}^{0}$ dalla legge fisiologica sull' evoluzione delle vocali $e \hat{e} i ;$ e $2^{0}$ dal conguagliamento delle quantità latine nella evoluzione italiana; e dimostrare come questi due fatti accoppiati non si possano spiegare se non nell' ipotesi che l' $\frac{f}{e}$ abbia veramente suonato larga, e $l^{\prime} e^{t}$ stretta. Il modo diverso in 
cui i avvenuto il conguagliamento delle quantità d un effetto delle diverse abitudini glottiche dei popoli che appresero il latino.

F. come si spiegano $i$ pochi casi schiettamente toscani di $i$ da 2 ? Le vie sarebbero parecchie; e ci basta accennare come la più naturale quella, che $i$ loro temi latini avessero il suono chiuso invece dell' aperto: e così da un racémum si potè venire, coll' evoluzione regolare del mezzo tuono, a racimo-lo ecc. Qualche esemplare latino, in cui la grafia oscilla fra l' $\bar{e}$ e l'oe perfino nelle iscrizioni, ci assicura che eccezioni poterono ben esservi anche nel latino classico alla proferenza larga dell' $\bar{e}$. Ma non furono per certo numerose. - Gli esemplari italiani con $i$ più abbondano in sillaba aperta che in posizione: e questo servirà a confermarci nella credenza, che la posizione non abbia mai abbreviata la vocale, la quale, in tal caso, avrebbe dovuto maggiormente restringersi, e arrivare più facilmente all' $i$.

L' é breve latina, nell' allungarsi, accolse dopo di se un elemento di $e$ proferito con lingua meno innalzata, ossia di $e$; e cosi dal lat. ténet s' ebbe té̀ $n e$ indi, poggiando l' accento sul secondo elemento onde conservare il posto originario, téene, alfine tiène, col dittongo $i \ell$; in cui il primo elemento ha una durata eguale alla metà del secondo; rappresentando così l' è il lat. $\breve{e}$, e l' $i$ l' aggiunta quantitativa italiana. L' $i$ poi si svolse dall' $\dot{\epsilon}$ dinanzi a vocale, come in mie da méae ecc. $(\$ \mathrm{X} b$ ). - Questo elemento $i$ del dittongo raccolto potè anche non svilupparsi, o, sviluppatosi, rifondersi con l' $e$ onde sorgeva, come in bene trèma: e ciò avvenne specialmente là dove per il nesso delle consonanti attigue diventava o difficile o impossibile alla glottide italiana di proferirlo. In fatto nella posizione ordinaria l' iè non si vede: sèsto pèste, cfr. lo sp. siesta; e nella posizione debole solo allora si mostra, quando il nesso che segue fa sillaba interamente con altra vocale: pie-tra, die-tro; e non fiebre ma feb-bre. Così quandu la posizione, per cosi dire, precedeva, tacque il dittongo: trema prèga ecc.

U. A. Cankllo. 